\title{
Structure et diversité floristique des îlots de forêts riveraines communautaires de la Basse vallée de la Sô au Sud-Est du Bénin
}

\author{
Jacques Boco ADJAKPA1, Hounnankpon YEDOMONHAN2, Léonard E. AHOTON 3 , Peter D. M.
} WEESIE4 \& Léonard E. AKPO5

'Université d'Abomey-Calavi, École Polytechnique d'Abomey-Calavi, Laboratoire de Rechercher en Biologie Appliquée, 01 BP 2009 Cotonou (Bénin),

2 Université d'Abomey-Calavi, Faculté des Sciences et Techniques, Laboratoire de Botanique et Écologie Végétale, Abomey-Calavi,( Bénin)

3Université d'Abomey-Calavi, Faculté des Sciences Agronomiques, Abomey-Calavi, (Bénin)

4University of Groningen, Faculty of Mathematics and Natural Sciences, Science and Society Group, (The Nederlands)

5Université Cheikh Anta Diop, Faculté des Sciences et Techniques, Laboratoire d'Ecologie et d'Ecohydrologie, Dakar (Sénégal)

Correspondant auteur: adjakpaj@yahoo.fr

Original submitted in on $11^{\text {th }}$ November 2012 Published online at www.m.elewa.org on 29th May 2013.

http://dx.doi.org/10.4314/jab.v65i0.89573

\section{RESUME}

Objectif : L'étude a pour objectif d'évaluer la structure et la diversité floristique des îlots de forêts riveraines communautaires de la basse vallée de la Sô au Sud-Est du Bénin.

Méthode et résultats: Les inventaires floristiques et forestiers ont été réalisés à travers 38 placeaux. Au total, 75 espèces réparties en 69 genres et 31 familles sont inventoriées. Les groupements végétaux identifiés et caractérisés sont la forêt riveraine à Mimusops kummel et Pterocarpus santalinoides, la forêt riveraine à Cola gigantea et $C$. laurifolia et la forêt riveraine à Berlinia grandiflora et Dialium guineense. Les paramètres floristiques et structuraux varient d'un groupement à l'autre. Ainsi, la richesse spécifique varie de 30 à 47 espèces, l'indice de diversité de Shannon de 1,65 à 4,20 bits, l'équitabilité de Pielou de 0,50 à 0,83 et la diversité des familles est comprise entre 19 et 24 avec une prédominance des Leguminosae. La densité varie de 247 à 641 tiges/ha, la surface terrière de 22,25 à 63,84 $\mathrm{m}^{2} / \mathrm{ha}$, le volume et la biomasse sont compris entre 85,62 et $471,01 \mathrm{~m}^{3} / \mathrm{ha}$.

Conclusion et application: L'étude a permis de montrer la diversité des groupements végétaux de ces forêts communautaires. Ces dernières augmentent localement la diversité biologique mais elles sont soumises à une forte pression anthropique. Ceci nécessite des actions urgentes de conservation. La protection de ces écosystèmes fragiles est primordiale pour le Sud du Bénin dans la mesure où ils constituent une zone humide d'importance internationale (site Ramsar 1018) et un potentiel économique pour les populations riveraines lacustres.

Mots clés : Flore, groupement, Basse vallée de la Sô, Sud-Est, Bénin. 


\section{Structural and floristic diversity of the riparian forest in the Sô lower valley in Benin Abstract}

Objective: This study aims to evaluate the structure and floristic diversity of the forest bordering the lower valley of the Sô southeast of Benin.

Methods and Results: The floristic inventories and forestry products were conducted across 38 plots. In total, 75 species belonging to 69 genera and 31 families were surveyed. Plant communities were identified and characterized as the riparian forest to Mimusops kummel and Pterocarpus santalinoides, riparian forest Cola gigantea and C. laurifolia and riparian forest to Berlinia grandiflora and Dialium guineense. Plant and structural parameters varied from one group to another. Thus, species richness varies from 30 to 47 species, Shannon diversity index of 1.65 to 4.20 bits, Pielou evenness from 0.50 to 0.83 and the diversity of families is between 19 and 24 with a predominance of Leguminosae. Density varies from 247 to 641 stems / ha, basal area of 22.25 to $63.84 \mathrm{~m}^{2}$ / ha, volume and biomass are between 85.62 and $471.01 \mathrm{~m} 3 / \mathrm{ha}$.

Conclusion and application: The study showed the diversity of plant community forests. There is a recent increase in local biodiversity, but is subject to strong human pressure which requires urgent conservation actions. The protection of these fragile ecosystems is crucial for southern Benin since it constitute a wetland of international importance (Ramsar 1018) and economic potential for the populations living on the lakeside.

Keywords : Flora, community, Sô lower valley, South-East of Benin.

\section{INTRODUCTION}

Les forêts tropicales sont des écosystèmes extrêmement utiles et précieux. Elles jouent un rôle capital dans la régulation de l'effet de serre, dans les grands équilibres climatiques et constituent le plus grand réservoir de biodiversité de la planète (Tchatat, 1999). Les forêts limitent le ruissellement des eaux de pluies en favorisant leur infiltration. Elles protègent les sols contre l'érosion, améliorent le micro-climat en réduisant la température au niveau du sol et assurent le maintien des qualités nutritives du sol (Bergonzini \& Lanly, 2000). La conférence des Nations-Unies sur l'environnement et le développement tenue à Rio en 1992 a reconnu que ce sont les activités anthropiques qui sont à la base de la dégradation

\section{MATERIEL ET METHODE}

Milieu d'étude : L'étude a été menée dans les îlots forestiers communautaires de la basse vallée de la Sô au Sud-Est du Bénin (figure 1). Ces îlots sont localisés à Ahomey-Lokpo $\left(06^{\circ} 34^{\prime} 59^{\prime \prime} \mathrm{N}\right.$ et $\left.002^{\circ} 24^{\prime} 19^{\prime \prime} \mathrm{E}\right)$ dans la commune de So-Ava, à Fandji $\left(06^{\circ} 39^{\prime} 06^{\prime \prime} \mathrm{N}\right.$ et $002^{\circ} 23^{\prime} 19^{\prime \prime}$ E) dans la commune d'Abomey-Calavi et à Togbota $\left(06^{\circ} 41^{\prime} 59^{\prime \prime} \mathrm{N}\right.$ et $\left.002^{\circ} 25^{\prime} 05^{\prime \prime} \mathrm{E}\right)$ dans la commune d'Adjohoun. La zone d'étude jouit d'un climat subéquatorial caractérisé par l'alternance de deux des écosystèmes. Au Bénin, les pressions s'accentuent au jour le jour sur les ressources naturelles en général et forestières en particulier à cause de l'augmentation de la population et d'une mauvaise politique de gestion de ces ressources. Pour ce faire, avant la mise en place d'une politique de gestion participative et l'installation des plantations pour le bien être des générations futures, il est nécessaire de faire un inventaire judicieux de la biodiversité contenue dans les îlots forestiers restants. C'est dans ce cadre que s'inscrit notre travail qui a pour objectif d'étudier la diversité floristique et la structure des îlots de forêts riveraines communautaires de la basse vallée de la Sô au Sud-Est du Bénin.

saisons sèches et deux saisons pluvieuses très marquées. La pluviosité moyenne annuelle est de 1346,6 mm. La moyenne annuelle des températures est de $27,5^{\circ} \mathrm{C}$ (Adjakpa, 2006). La saison pluvieuse dure 7 mois (mi-mars à juillet et mi-septembre à octobre). La saison sèche couvre les mois de novembre à mi-mars et d'août à mi-septembre. Le milieu est une plaine côtière, basse et sableuse, marécageuse par endroits, appartenant au système 


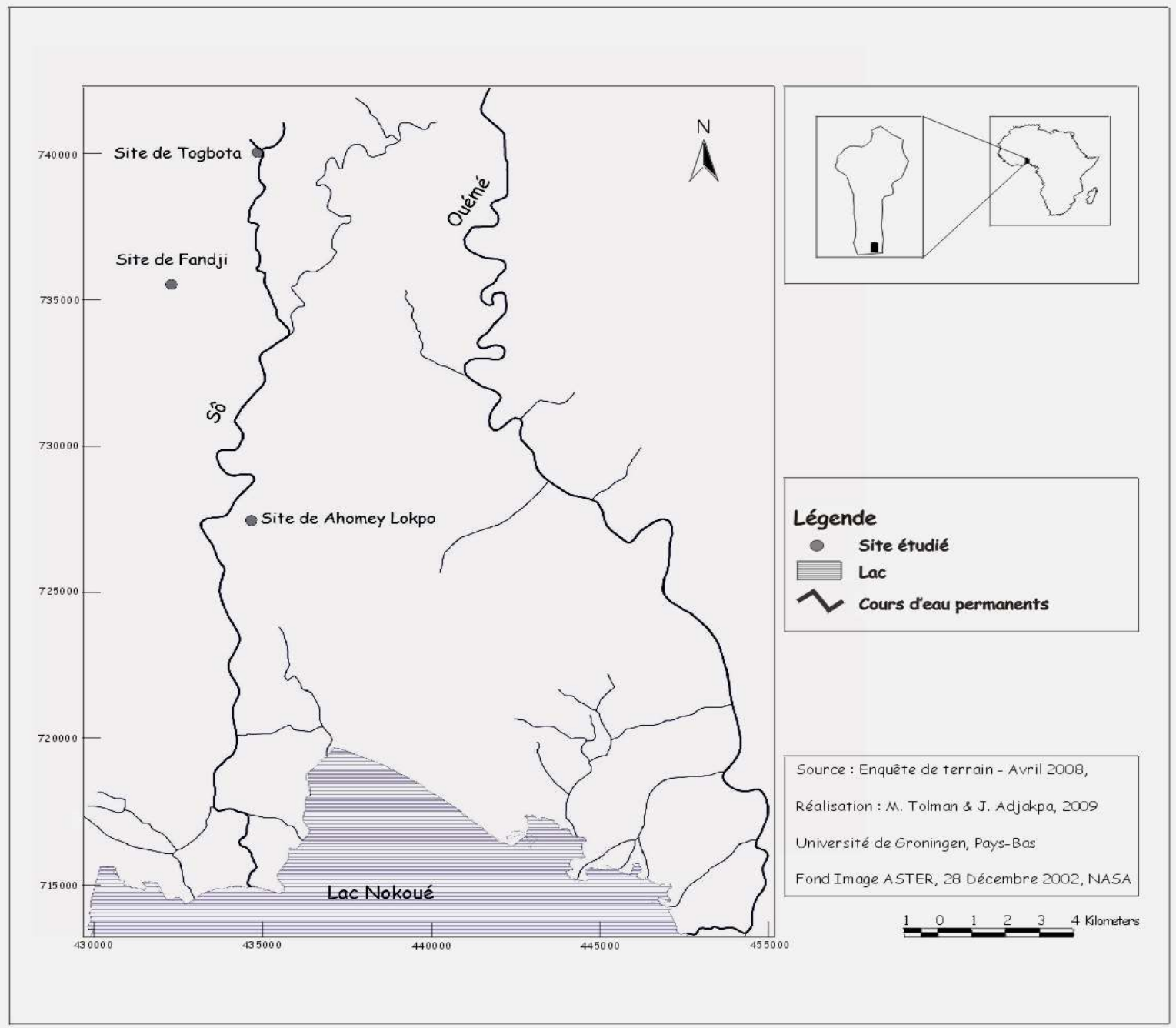

Figure 1: Localisation des sites étudiés

\section{Méthode}

Collecte des données: Les données ont été collectées entre mars et avril 2008. Au total, 38 placeaux ont été installés. Les inventaires floristiques ont été effectués dans 6 îlots de forêts communautaires dont 4 à Ahomey-Lokpo, 1 à Fandji et 1 à Togbota. Au niveau de chaque point d'inventaire, deux placeaux circulaires concentriques de $18 \mathrm{~m}$ et de $5 \mathrm{~m}$ de rayon ont été installés. Ensuite, cinq placeaux carrés de $2 \mathrm{~m}$ de côté, dont un au centre des cercles et quatre aux extrémités de deux diamètres obliques du cercle de 18 $\mathrm{m}$ de rayon sont installés. Dans les placeaux circulaires de $18 \mathrm{~m}$ de rayon, les mesures dendrométriques (diamètre, hauteur fût et hauteur totale) sont prises pour toutes les espèces ligneuses dont le diamètre à hauteur de poitrine d'homme (d.b.h.) est supérieur à $7,5 \mathrm{~cm}$. A l'intérieur des placeaux circulaires de $5 \mathrm{~m}$ de rayon, les arbustes et jeunes arbres de d.b.h. compris entre 3 et $7,5 \mathrm{~cm}$ sont comptés en vue de déterminer leur densité. Les placeaux carrés de $2 \mathrm{~m}$ de côté ont permis de recenser les ligneux à d.b.h. inférieur à $3 \mathrm{~cm}$ avec leur coefficient d'abondance-dominance suivant les échelles de Braun-Blanquet (1932). Les espèces non identifiées sur le terrain ont été échantillonnées et déterminées par la suite à l'Herbier National du Bénin par comparaison aux spécimens de références.

Traitement des données : Le tableau de contingence en présence-absence des espèces a été établi avec le tableur Excel à partir des données de relevés floristiques. II a été soumis à la classification 
hiérarchique à l'aide du logiciel Community Analysis Package (CAP) (Hendenson \& Seaby, 2002) qui a permis la partition des relevés en groupements végétaux. La matrice relevés-espèces a ensuite servi à évaluer la diversité floristique et établir les spectres écologiques (spectre biologique brut et spectre phytogéographique) par groupements végétaux identifiés et pour l'ensemble des îlots forestiers. La diversité floristique des différents groupements végétaux a été évaluée à l'aide de la richesse spécifique, de la diversité en genres et de celle en familles (Daget, 1980). La nomenclature botanique utilisée est celle de la Flore Analytique du Bénin (Akoègninou et al. 2006). Les spectres biologiques bruts ont été construits à partir des types biologiques de Raunkiaer (1934) et les spectres phytogéographiques ont été établis à l'aide des types phytogéographiques définis par White (1986). La structure du peuplement arborescent (d.b.h. $\geq 7,5 \mathrm{~cm}$ ) a été évaluée à l'aide de la surface terrière, la densité, le volume, la biomasse, l'indice de diversité de Shannon et l'équitabilité de Pielou. La surface terrière du peuplement $(G)$ est la somme des sections des troncs d'arbres (d.b.h. $\geq 7,5 \mathrm{~cm}$ ). Elle est obtenue par la formule :

\section{$G=\Sigma \pi D^{2} / 4$}

Avec $\mathrm{D}=$ diamètre à hauteur de poitrine d'homme des arbres.

La densité du peuplement $(\mathrm{N})$ correspond au nombre de tiges à l'hectare.

Le volume $(\mathrm{V})$ et la biomasse $(\mathrm{B})$ sont calculés suivant les formules de Dawkins (1961):

$V=\Sigma \pi D^{2} / 4 \times H \times 0,555$ et $B=D^{2} \times H \times 0,283$

avec $\mathrm{H}$ la hauteur totale ou la hauteur fût, $\mathrm{D}=$ diamètre à hauteur portion d'hommes,

\section{RESULTATS}

Typologie des formations végétales: La classification hiérarchique individualise 3 groupes de relevés assimilés à 3 groupements végétaux (figure 2) à savoir :

- le groupe de relevés désigné GI qui représente la forêt riveraine à Mimusops kummel et Pterocarpus santalinoides (Photo 1),
L'indice de diversité de Shannon $(\mathrm{H})$ est donné par la formule :

\section{$H=-\Sigma\left[\left(N_{i} / N\right) \log 2\left(N_{i} / N\right)\right]$,}

Avec $\mathrm{N}_{\mathrm{i}}$ la densité de l'espèce $\mathrm{i}$ et $\mathrm{N}$ la densité de toutes les espèces de la communauté.

La diversité est faible lorsque $\mathrm{H}$ est inférieur à 3 bits, moyenne si $\mathrm{H}$ est compris entre 3 et 4 puis élevé quand $\mathrm{H}$ est supérieur ou égal à 4 bits (Legendre \& Legendre, 1984 ; Frontier \& Pichod-Viale, 1995). L'équitabilité de Pielou (E) traduit la manière dont les individus sont distribués à travers les espèces. Elle est maximale si les individus sont répartis de la même manière à travers les espèces. Elle varie de 0 (une espèce a une très forte abondance) à 1 (toutes les espèces ont la même importance). Elle se calcule par la formule suivante :

\section{$E=H / \log _{2}(R s)$,}

Où Rs désigne la richesse spécifique et $\mathrm{H}$ l'indicée de divernu de Shannon (Pielou, 1996)

L'indice de valeur d'importance des espèces (IVIE) de Cottam \& Curtis (1956) a été calculé suivant la formule :

\section{$I V I E=100^{\star}\left[\left(N_{i} / \Sigma N_{i}\right)+\left(G_{i} / \Sigma G_{i}\right)+\left(F_{i} / \Sigma F_{i}\right)\right]$}

Avec $\mathrm{N}_{\mathrm{i}}=$ nombre d'individus de l'espèce $\mathrm{i}, \mathrm{G}_{\mathrm{i}}=$ surface terrière de l'espèce $\mathrm{i}, \mathrm{F}_{\mathrm{i}}=$ fréquence de l'espèce $\mathrm{i}$. Les espèces à IVIE $\geq 10$ sont celles écologiquement importantes (Reitsma, 1988).

La densité de la régénération a été aussi évaluée par groupement végétal.

- le groupe GII constitué de relevés exécutés dans la forêt riveraine à Cola gigantea et Cola laurifolia (Photo 2),

- le groupe GIII qui comporte les relevés effectués dans la forêt riveraine à Berlinia grandiflora et Dialium guineense (Photo 3 ) 


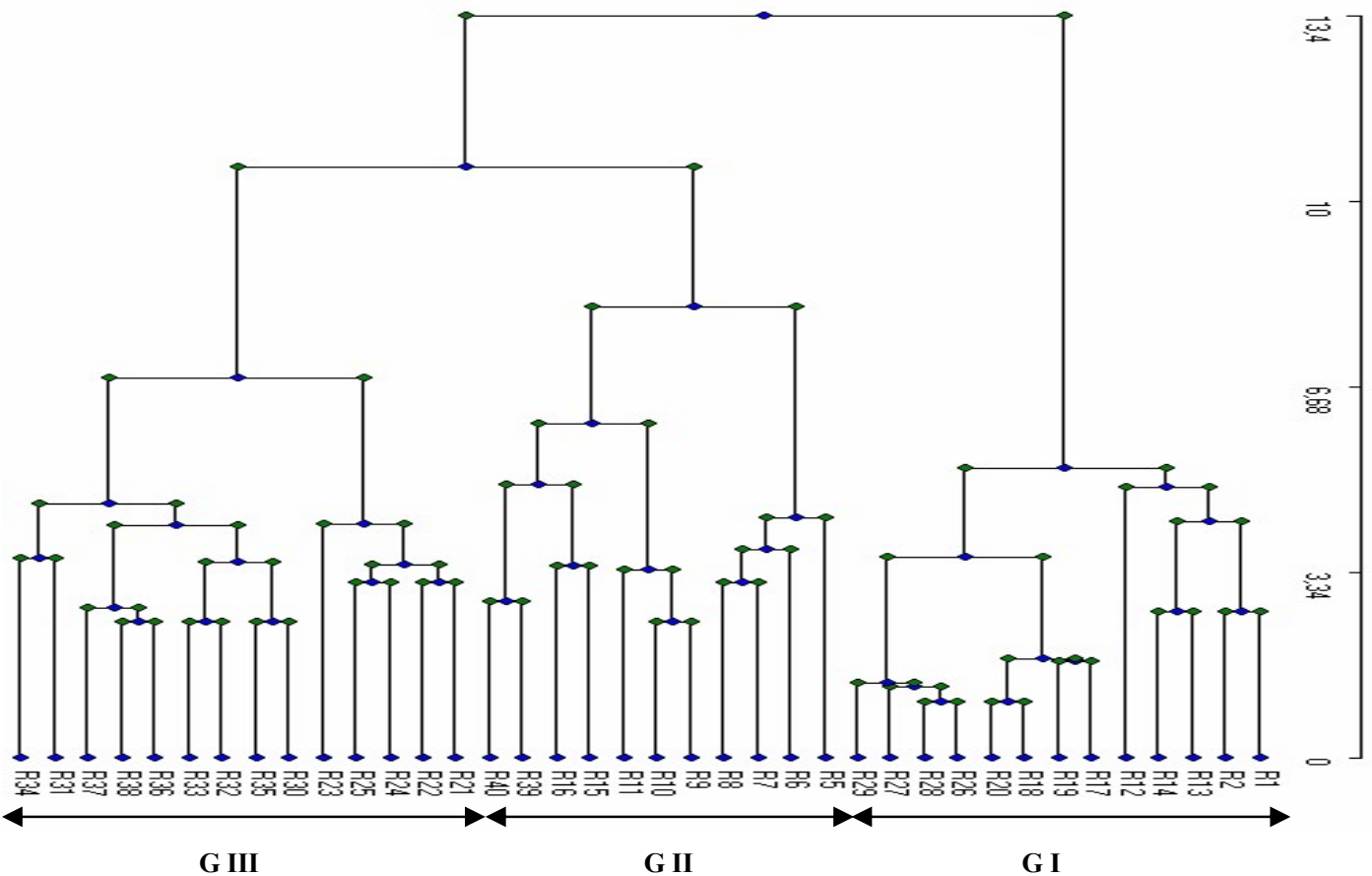

Figure 2 : Partition des groupements végétaux des forêts riveraines communautaires de la basse vallée de la Sô (Gl : forêt riveraine à Mimusops kummel et Pterocarpus santalinoides, GIl : forêt riveraine à Cola gigantea et $C$. laurifolia, GIII : Forêt riveraine à Berlinia grandiflora et Dialium guineense)

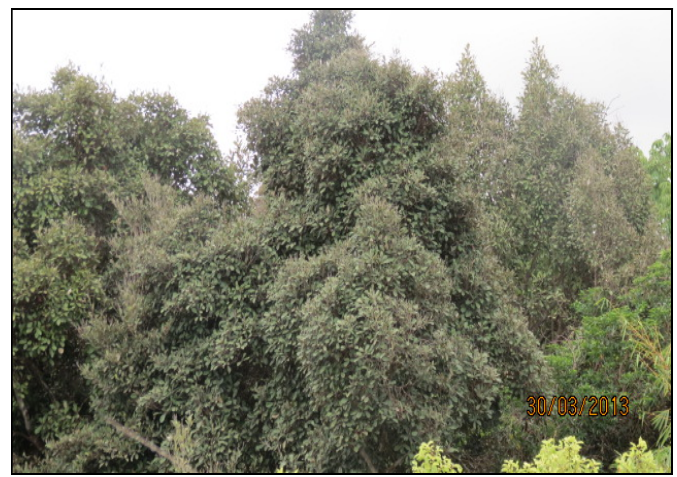

Photo 1 : Forêt riveraine à $M$. kummel et $P$. santalinoides

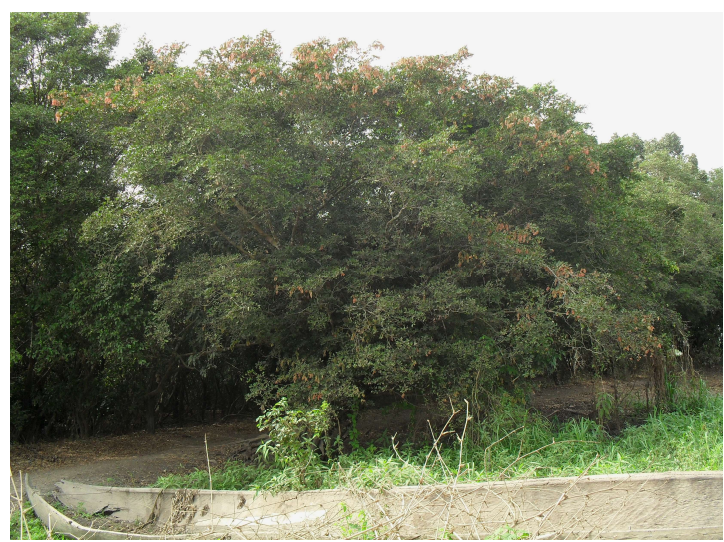

Photo 3 : Forêt riveraine à $B$. grandiflora et $D$. guineense

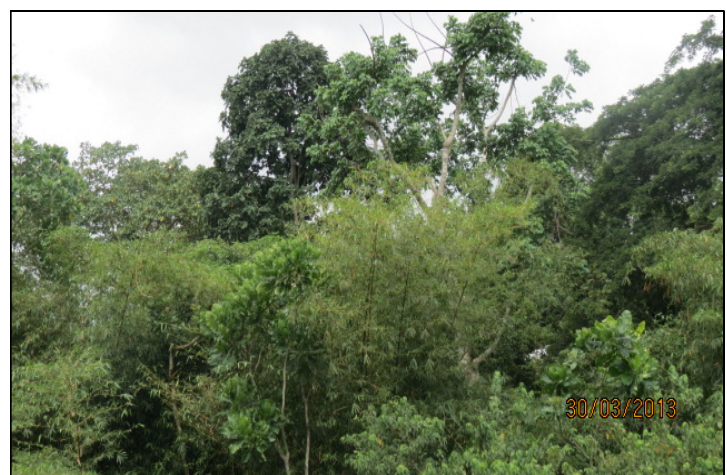

Photo 2 : Forêt riveraine à C. gigantea et C. laurifolia 
Diversité floristique: Au total, 75 espèces réparties en 69 genres et 31 familles ont été recensées (annexe 1). Le tableau 1 présente la diversité floristique des groupements. La forêt riveraine à Cola gigantea et $C$. laurifolia est la plus diversifiée en espèces (47), en genres (44) et en familles (24). Par contre, le groupement à Mimusops kummel et Pterocarpus santalinoides est celui le plus pauvre sur le plan floristique avec (30 espèces, 29 genres et 19 familles). Les Leguminosae constituent la principale famille de plantes, avec 10 genres (14,69\%) et 11 espèces $(14,67 \%)$. Les Rubiaceae, avec 8 genres $(11,59 \%)$ et 9 espèces (12\%) représentent la deuxième famille la plus diversifiée. Ensuite, viennent les Euphorbiaceae et les Sapindaceae avec chacune 5 genres $(7,25 \%)$ et 5 espèces $(6,67 \%)$. Tous les 3 groupements végétaux sont caractérisés par la prédominance, en terme de richesse spécifique, des Leguminosae (10,64 à $15,79 \%$ ) suivies des Rubiaceae (10 à 13,16\%).

Tableau 1 : Diversité floristique des groupements végétaux

\begin{tabular}{|c|c|c|c|c|}
\hline Groupements végétaux & $\begin{array}{l}\text { Richesse } \\
\text { spécifique }\end{array}$ & $\begin{array}{l}\text { Nombre } \\
\text { de genres }\end{array}$ & $\begin{array}{c}\text { Nombre } \\
\text { de } \\
\text { familles }\end{array}$ & $\begin{array}{l}\text { Familles dominantes en terme de } \\
\text { richesse spécifique }\end{array}$ \\
\hline $\begin{array}{l}\text { Forêt riveraine à Mimusops } \\
\text { kummel et Pterocarpus } \\
\text { santalinoides }\end{array}$ & 30 & 29 & 19 & $\begin{array}{c}\text { Leguminosae }(13,38 \%) \text {, Euphorbiaceae } \\
\text { et Rubiaceae (10\% chacune) }\end{array}$ \\
\hline $\begin{array}{l}\text { Forêt riveraine à Cola gigantea } \\
\text { et } C \text {. laurifolia }\end{array}$ & 47 & 44 & 24 & $\begin{array}{c}\text { Leguminosae et Rubiaceae }(10,64 \% \\
\text { chacune), Apocynaceae et Sapindaceae } \\
(8,51 \% \text { chacune })\end{array}$ \\
\hline $\begin{array}{l}\text { Forêt riveraine à Berlinia } \\
\text { grandiflora et Dialium } \\
\text { guineense }\end{array}$ & 38 & 36 & 23 & $\begin{array}{c}\text { Leguminosae }(15,79 \%), \text { Rubiaceae } \\
(13,16 \%), \text { Sapindaceae }(10,53 \%)\end{array}$ \\
\hline Total & 75 & 69 & 31 & $\begin{array}{c}\text { Leguminosae }(14,67 \%) \text {, Rubiaceae } \\
(12 \%), \text { Euphorbiaceae et Sapindaceae } \\
(6,67 \% \text { chacune })\end{array}$ \\
\hline
\end{tabular}

Spectre biologique: De façon globale, les microphanérophytes représentent les types biologiques les plus fréquents (34 espèces) soit $45 \%$ dont $11 \%$ de lianes. Ils sont suivis des mésophanérophytes $(23$ espèces) soit $31 \%$ dont $1 \%$ de lianes, des nanophanérophytes (15 espèces) soit $20 \%$ dont $3 \%$ de lianes et des mégaphanérophytes (3 espèces). La figure 3 montre que les microphanérophytes constituent les types biologiques majoritaires au niveau de la forêt riveraine à Cola gigantea et $C$. laurifolia, avec un taux de $53,19 \%$ et de la forêt riveraine à Berlinia grandiflora et Dialium guineense (42,11\%). Par contre, dans la forêt riveraine Mimusops kummel et Pterocarpus santalinoides, ceux sont les mésophanérophytes qui prédominent avec $46,67 \%$.
Spectres phytogéographiques: Les espèces guinéennes prédominent avec un taux de 59\% dont $56 \%$ d'espèces guinéo-congolaises et $3 \%$ d'espèces guinéo-orientales. Les espèces plurirégionales représentent $15 \%$. Les espèces soudaniennes font $27 \%$ dont $22 \%$ d'espèces soudano-guinéennes et $5 \%$ d'espèces. La flore guinéenne est largement majoritaire dans la forêt riveraine à Berlinia grandiflora et Dialium guineense et dans la forêt riveraine à Cola gigantea et C. laurifolia avec respectivement un taux de 63 et $68 \%$ (figure 4). Par contre, elle est relativement mais importante $(37 \%)$ dans la forêt riveraine à Mimusops kummel et Pterocarpus santalinoides où les espèces soudaniennes font $40 \%$ dont $27 \%$ pour les soudanoguinéennes et $13 \%$ pour les soudano-zambésiennes. 


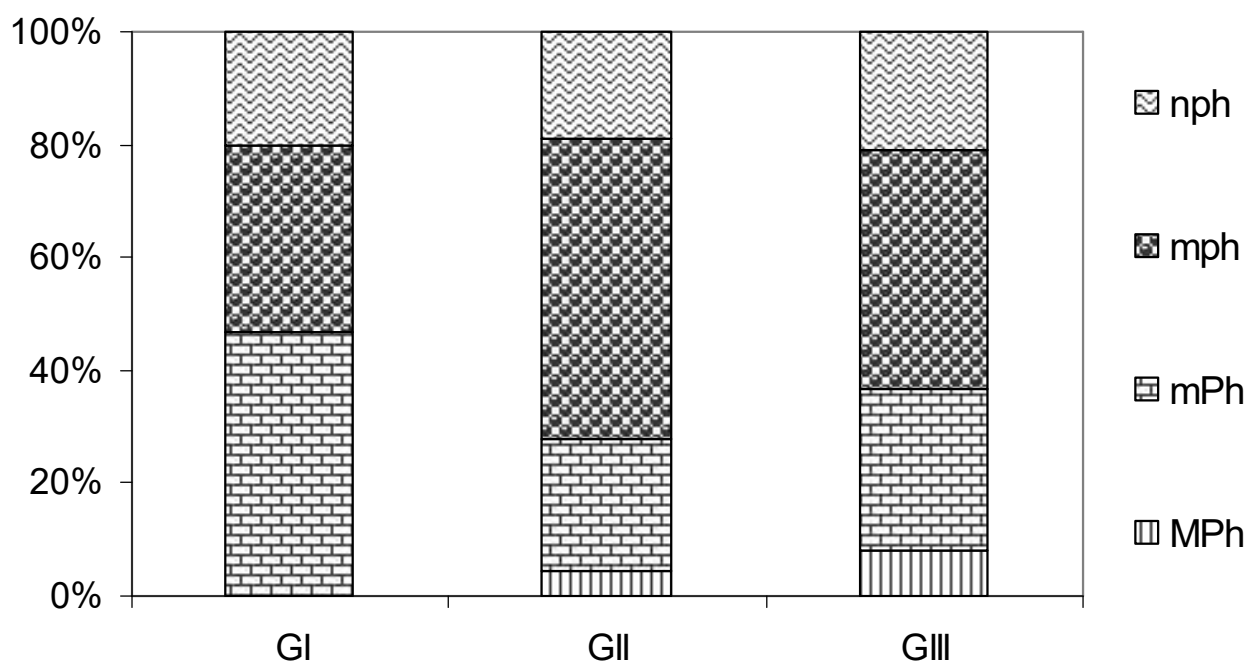

Figure 3 : Spectres biologiques des trois groupements végétaux $\mathrm{GI}$ : forêt riveraine à Mimusops kummel et Pterocarpus santalinoides, GIl : forêt riveraine à Cola gigantea et $C$. laurifolia, GIII : forêt riveraine à Berlinia grandiflora et Dialium guineense, MPh : mégaphanérophytes, mPh : mésophanérophytes, mph : microphanérophytes, nph : nanophanérophytes

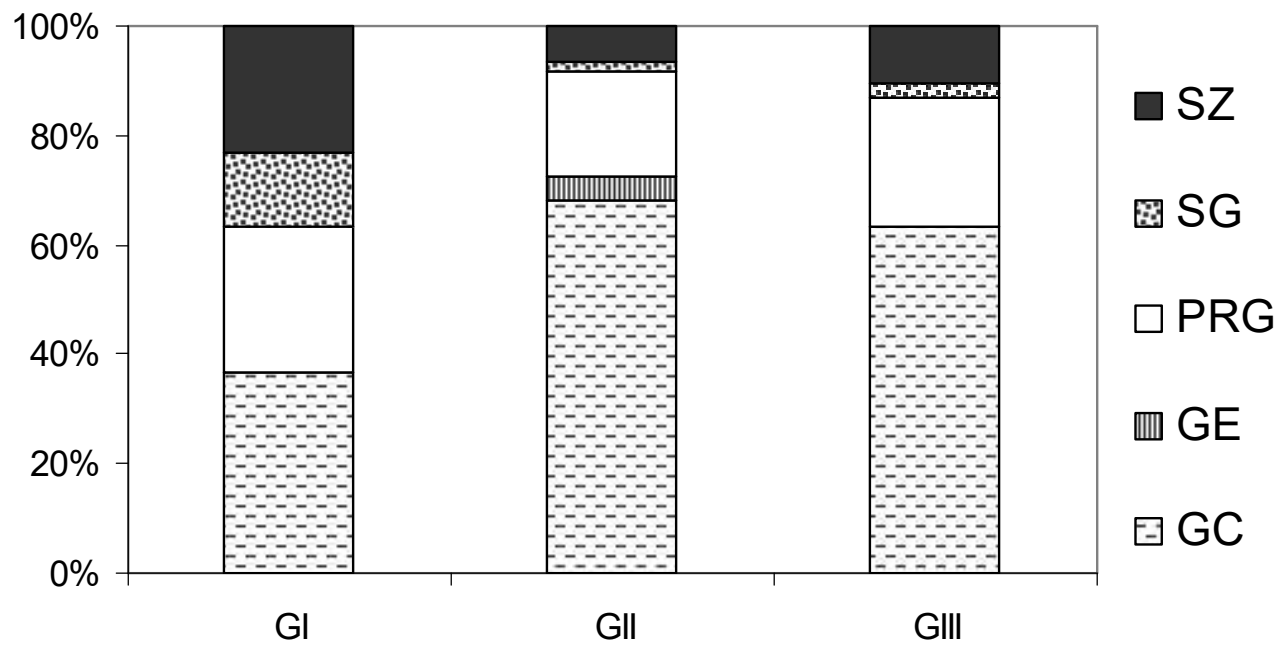

Figure 4 : Spectres phytogéographiques des trois groupements végétaux $\mathrm{Gl}$ : forêt riveraine à Mimusops kummel et Pterocarpus santalinoides, GIl : forêt riveraine à Cola gigantea et $C$. laurifolia, GIII : forêt riveraine à Berlinia grandiflora et Dialium guineense, GC : espèces guinéo-congolaises, GE : espèces guinéo-orientales, SG : espèces soudano-guinéennes, SZ : espèces soudano-zambéziennes, PRG : espèces plurirégionales.

Caractéristiques structurales des peuplements ligneux: La surface terrière, la densité, l'indice de diversité de Shannon, l'équitabilité de Pielou, la biomasse et le volume des peuplements ligneux sont présentés dans le tableau 2. La surface terrière moyenne varie de $22,15 \mathrm{~m}^{2} /$ ha dans la forêt riveraine à Cola gigantea et Cola laurifolia à $63,84 \mathrm{~m}^{2} /$ ha dans la forêt riveraine à Mimusops kummel et Pterocarpus santalinoides, avec une moyenne de $38,66 \mathrm{~m}^{2} / \mathrm{ha}$. La densité est de 449 tiges/ha en moyenne. Elle très élevée (641 tiges/ha) dans la forêt riveraine à Mimusops kummel et Pterocarpus santalinoides puis plus faible (247 tiges/ha) dans le groupement à Berlinia grandiflora et Dialium guineense.

L'indice de diversité de Shannon varie de 1,65 à 4,20 bits, avec une moyenne de 3,01 bits. L'équitabilité de 
Pielou est comprise entre 0,50 et 0,83 . Le volume total et la biomasse totale sont respectivement compris entre 131,95 et $243,44 \mathrm{~m}^{3} /$ ha puis 85,62 et 471,02 $\mathrm{kg} / \mathrm{ha}$. La densité de régénération varie de 1233 à 4943 tiges/ha, avec une moyenne de 3361 tiges/ha.

Tableau 2 : Caractéristiques structurales des peuplements ligneux

\begin{tabular}{|c|c|c|c|c|c|c|c|}
\hline Groupements végétaux & $\mathbf{G}\left(\mathrm{m}^{2} / \mathrm{ha}\right)$ & $\mathbf{N}$ (tiges/ha) & H (bits) & $E$ & Volume ( $\left.\mathrm{m}^{3} / \mathrm{ha}\right)$ & $\begin{array}{l}\text { Biomasse } \\
\text { (kg/ha) }\end{array}$ & $\begin{array}{l}\text { Régénération } \\
\text { (tiges/ha) }\end{array}$ \\
\hline $\mathrm{GI}$ & 63,84 & 641 & 1,65 & 0,50 & 243,44 & 471,02 & 1233 \\
\hline GIl & 22,15 & 458 & 4,20 & 0,83 & 177,13 & 244,95 & 4943 \\
\hline GIII & 30 & 247 & 3,17 & 0,67 & 131,95 & 85,62 & 3906 \\
\hline Moyenne & $\begin{array}{c}38,66 \\
\pm \\
22,15\end{array}$ & $\begin{array}{c}449 \\
\pm \\
197\end{array}$ & $\begin{array}{c}3,01 \\
\pm \\
1,28\end{array}$ & $\begin{array}{c}0,67 \\
\pm \\
0,17\end{array}$ & $\begin{array}{c}184,17 \\
\pm \\
56,08\end{array}$ & $\begin{array}{c}267,19 \\
\pm \\
193,66\end{array}$ & $\begin{array}{c}3361 \\
\pm \\
1914\end{array}$ \\
\hline
\end{tabular}

$\mathrm{Gl}$ : Forêt riveraine à Mimusops kummel et Pterocarpus santalinoides, GIl : Forêt riveraine à Cola laurifolia et $\mathrm{C}$. gigantea, GIII : Forêt riveraine à Berlinia grandiflora et Dialium guineense, G : Surface terrière, $\mathrm{N}$ : densité, $\mathrm{H}$ : indice de diversité de Shannon, $\mathrm{E}$ : équitabilité de Pielou

Les îlots de forêt riveraine étudiés renferment 6 espèces qui ont un IVIE $\geq 10$ (tableau 3). Ces dernières représentent alors les espèces écologiquement importantes. II s'agit, dans l'ordre décroissant, de : Elaeis guineensis qui a un IVIE de
48, Acacia auriculiformis (40), Berlinia grandiflora (27), Dialium guineense (24), Hura crepitans (12) et Mimusops kummel (10). Les deux premières espèces ayant de grande importance écologique sont des espèces plantées.

Tableau 3 : Liste des espèces de grande importance écologique du peuplement arborescent

\begin{tabular}{lcccc}
\hline Espèces & $\begin{array}{c}\text { Densité relative } \\
(\mathbf{\%})\end{array}$ & $\begin{array}{c}\text { Fréquence relative } \\
(\mathbf{\%})\end{array}$ & $\begin{array}{c}\text { Surface terrière } \\
\text { Relative (\%) }\end{array}$ & IVIE \\
\hline Elaeis guineensis & 9,34 & 25,5 & 13 & 48 \\
Acacia auriculiformis & 32,3 & 1,88 & 6,1 & 40 \\
Berlinia grandiflora & 6,6 & 3,98 & 16 & 27 \\
Dialium guineense & 5,78 & 4,97 & 13 & 24 \\
Hura crepitans & 3,62 & 0,99 & 7,20 & 12 \\
Mimusops kummel & 4,67 & 1,79 & 3,54 & 10 \\
\hline
\end{tabular}

IVIE : Indice de Valeur d'Importance des espèces

\section{DISCUSSION}

La diversité floristique des forêts riveraines de la basse vallée de la Sô à l'Est du Bénin est de 75 espèces, 69 genres et 31 familles. Elle est une bonne contribution à la flore forestière du Bénin. Toutefois, elle reste nettement inférieure à celle de 120 espèces réparties en 47 familles pour l'ensemble des forêts riveraines du Bénin (Akoègninou, 2004) et à celle de 238 espèces, 203 genres et 75 familles obtenue par Adjakpa (2006) dans la basse vallée de l'Ouémé. Cette différence observée serait liée à la taille et au degré d'anthropisation des îlots forestiers. Les familles botaniques les plus riches en espèces sont les Leguminosae et les Rubiaceae. La prédominance des Leguminosae n'est pas une caractéristique des îlots étudiés mais une constante de la flore du Bénin (Akoègninou, 2006). S'agissant des Rubiaceae, la majorité des taxons appartenant à cette famille sont forestiers (Akoègninou, 2004 ; Adomou, 2005). Les travaux de Akoègninou (2004) ont abouti à la même conclusion, avec comme trois premières familles, les Leguminosae $(11 \%)$, les Rubiaceae $(8 \%)$ et les Euphorbiaceae $(5 \%)$. Les proportions des types biologiques montrent une prédominance des microphanérophytes au sein de deux groupements végétaux (42 et $53 \%$ ) et la prédominance des mésophanérophytes au niveau du troisième groupement avec un taux de $47 \%$. Ce résultat est similaire à ceux obtenus par Akoègninou (2004) et 
Adjakpa (2006). La prédominance des microphanérophytes est une caractéristique générale des îlots forestiers du Bénin (Adomou, 2005) et est certainement liée à la forte pression anthropique sur les ressources forestières (Gbaguidi, 1998).

Les études biogéographiques de Akoègninou (1984), Akpagana (1989), Bossou (2001) et Yédomonhan et al. (2008) ont montré que les spectres phytogéographiques sont fonction des types de formation, Cependant, nos résultats corroborent ceux d'Akoègninou (2004) et Adjakpa (2006) où l'on note, pour l'ensemble des îlots prospectés, une forte proportion des espèces guinéennes $(59 \%)$ et une faible proportion des espèces soudaniennes $(27 \%)$. Ceci prouve que la flore prospectée est guinéenne. Le faible pourcentage des espèces soudaniennes est dû au milieu très humide favorisant la flore guinéenne (Akoègninou, 2004). Paradis (1975) pense que ces espèces soudaniennes se seraient retrouvées dans cette zone à la faveur du fleuve Ouémé. D'après les travaux de Malaisse (1982), la surface terrière minimale des forêts denses humides est de $40 \mathrm{~m}^{2} / \mathrm{ha}$. Seul le groupement à Mimusops kummel et Pterocarpus

\section{CONCLUSION}

Cette étude a permis d'identifier trois groupements végétaux: la forêt riveraine à Mimusops kummel et Pterocarpus santalinoides, la forêt riveraine à Cola gigantea et Cola laurifolia et celle à Berlinia grandiflora et Dialium guineense. Ces groupements végétaux sont repartis suivant les gradients topographiques et hydromorphiques. La flore compte 75 espèces parmi

\section{REMERCIEMENTS}

Cette étude a bénéficié d'un appui financier de I'Université de Groningen (Royaume des Pays-Bas) et du Centre de Recherche Ornithologique et de

\section{REFERENCES BIBLIOGRAPHIQUES}

Adjakpa BJ, 2006. Flore et végétation actuelle du Bas delta de l'Ouémé des zones humides du SudBénin. Thèse de Doctorat 3ème Cycle en Biologie végétale, Option Ecologie. Faculté des Sciences et Techniques/UCAD, Dakar (Sénégal), $88 \mathrm{p}$.

Adomou CA, 2005. Vegetation patterns and environmental gradient in Benin : Implication for biogeography and conservation. $\mathrm{PhD}$ Thesis, Wageningen University, $136 \mathrm{p}$.

Akoègninou $A, 1984$. Contribution à l'étude botanique des îlots de forêts denses humides semi- santalinoides présente un peuplement ligneux dont l'aire basale dépasse largement cette valeur minimale. Cependant, ceci ne traduit pas une bonne structuration naturelle, mais il est simplement lié au fait que c'est dans ce groupement que figurent les nombreux pieds de Acacia auriculiformis plantés.

La densité des arbres varie de 247 à 641 tiges/ha. Elle est bien située dans la fourchette de 167 à 1947 tiges/ha pour les forêts néotropicales (Gentry, 1982).

Suivant les échelles de Frontier \& Pichod-viale (1995), les valeurs de l'indice de diversité de Shannon montrent que la forêt riveraine à Cola gigantea et $C$. laurifolia est fortement diversifiée en arbres et arbustes, le groupement à Berlinia grandifolia et Dialium guineense a une diversité moyenne puis le groupement à Mimusops kummel et Pterocarpus santalinoides est faiblement diversifié. Ce dernier est caractérisé par la prédominance d'une espèce sur les autres comme en témoigne sa faible équitabilité de Pielou qui est de 0,50 . Quant à la régénération, sa forte densité témoigne de la bonne reconstitution des peuplements si ces derniers ne subissent pas trop de pressions anthropiques.

lesquelles figurent plusieurs espèces menacées de disparition telles Mimusops kummel, Cynometra vogelii, Dialum guineense, etc. Les trois importantes familles sont les Leguminosae, les Rubiaceae et les Euphorbiaceae. Les paramètres structuraux indiquent que malgré leur caractère sacré, les îlots étudiés ne sont pas à l'abri des pressions anthropiques.

l'Environnement (CEROE) à qui les auteurs expriment leur profonde gratitude.

décidues en République populaire du Bénin. Thèse du $3^{e}$ cycle, Université de Bordeaux III.250 p.

Akoègninou $A, 2004$. Recherches botaniques et écologiques sur les forêts naturelles du Bénin (Afrique de l'Ouest). Thèse d'Etat Université de COCODY-ABIDJAN 314p.

Akoègninou $A$, van der Burg, van der Maesen LJG, 2006. Flore analytique du Bénin. Backhuys Publishers, Cotonou \& Wageningen, $1032 \mathrm{p}$. 
Akpagana K, 1989. Recherches sur les forêts denses humides du Togo. Thèse Doctorat, Univ. Bordeaux III, 181p.

Bergonizi JC et Lanly JP, 2000. Les forêts tropicales. Paris, Kartalan, Cirad, $166 \mathrm{p}$.

Bossou B, 2001. Contribution à l'aménagement de la forêt classée de Dogo-Kétou: structure et dynamique des différents groupements végétaux et périodicité des coupes. Mémoire de DEA /FSA/UAC, Abomey-Calavi. Bénin.

Braun-Blanquet J, 1932. Plant sociology. The study of plant communities translated revised and edited by FULLER G.D. and CONARD. M. S.

Cottam G. et Curtis JT, 1956. The use of distance measurements in phytosociological sampling. Ecology 37: 451-460.

Daget $P, 1980$. Le nombre de diversité de Hill, un concept unificateur dans la théorie de la diversité écologique. Acta Oecological/Oecol. Gener 1 (1) : 51-70.

Dawkins HC, 1961. Estimating total volume of some cariblean trees. Cariblean for. 22 (3-4): 62-63

Frontier S et Pichod-Viale D., 1995. Ecosystèmes: structure, fonctionnement, évolution. $2^{\mathrm{e}}$ Edition. Collection d'écologie 21: 287-311. Paris. Masson.

Gbaguidi F, 1998. Forêts sacrées et conservation de la biodiversité dans le département de l'Ouémé au Sud-est du Bénin. Thèse d'Ingénieur Agronome. FSA/UNB. Bénin. 164 p.

Gentry AH, 1982. Patterns of neotropical plant species diversity. In: M.K. Hecht, B. Wallace \& G.T. Prance (eds.) Evolutionary biology 15: 123135. New York, Plenum Press.

Hendenson PA et Seaby RM, 2002. Community Analysis Package 2.15 (CAP). Ltd, IRC House, Pennington, Lymington, SO41 8 GN, UK.

Legendre et Legendre $\mathrm{P}, 1984$. Ecologie numérique. Tome 2: La structure des données écologiques. Masson collection d'écologie $n^{\circ} 13$.

Le Barbe Ale G, Millet B, Texier H, Borel Y, Guadle R, 1993. Les ressources en eaux superficielles de la République du Bénin. Ed. ORSTOM. Coll. Monographies hydrologiques $n^{\circ} 11$. Paris/ France.

Malaise F, 1982. Comparaison de la structure ligneuse dans une série régressive de la région zambésienne : forêt dense sèche - forêt claire - savane boisée (Luiswishi, Shaba, Zaïre). Geo-Eco-Trop 6 (4) : 309-350.

Paradis G, 1975. Physionomie, composition floristique et dynamisme des formations végétales d'une partie de la basse vallée de l'Ouémé (Dahomey). Ann. Univ. Abidjan, sér. E, 8: 281315

Pielou EC, 1996. Species diversity and pattern in the study of ecological succession. Theor. Biol. 10: $370-383$

Raunkiaer C, 1934. The life Forms of Plants and Statistical Plant Geography : 632 pp. Oxford, Oxford University Press.

Reistma JM, 1988. Forest vegetation in Gabon. Tropenbos technical series 1, $142 \mathrm{p}$. The Netherlands : Tropenbos Foundation.

Tchatat M, 1999. Produits forestiers autres que le bois d'œuvre (PFAB): Place dans l'aménagement durable des forêts denses humides d'Afrique Centrale. Série FORAFRI, document 18, 95p.

White $F, 1986$. La végétation de l'Afrique. Mémoire accompagnant la carte de végétation de I'Afrique. UNESCO/AETFAT/UNSO ORSTOMUNESCO. (1996). $384 \mathrm{p}$.

Yédomonhan $H$, Hounadagba $C J$, Akoègninou $A$, van der Maesen LJG, 2008 : Structure et diversité floristique de la végétation des inselbergs du secteur méridional du Centre-Bénin. Syst. Geogr. PI. $78: 111-125$.

Annexe 1 : Liste floristique

\begin{tabular}{|c|c|c|c|c|c|c|}
\hline TB & TP & Familles & Espèces & GI & GII & GIII \\
\hline $\mathrm{mPh}$ & PRG & Leguminosae-Mimosoideae & Acacia auriculiformis A. Cunn. ex Benth. & 1 & & \\
\hline Lnph & GC & Connaraceae & Agelaea pentagyna (Lam.) Baill. & & 1 & \\
\hline Mph & GC & Leguminosae-Mimosoideae & Albizia glaberrima (Schumach. \& Thonn.) Benth. & & 1 & \\
\hline Mph & GC & Euphorbiaceae & Alchornea cordifolia (Schumach. \& Thonn.) Müll.Arg. & & 1 & 1 \\
\hline Nph & SZ & Annonaceae & Annona senegalensis Pers. & 1 & & \\
\hline $\mathrm{mPh}$ & SG & Loganiaceae & Anthocleista djalonensis A.Chev. & & & \\
\hline
\end{tabular}




\begin{tabular}{|c|c|c|c|c|c|c|}
\hline \multicolumn{3}{|c|}{$\begin{array}{l}\text { Adjakpa et al. J. Appl. Biosci. } 2013 . \\
\text { communautaires de la vallée de Sô du Benin }\end{array}$} & \multicolumn{4}{|c|}{ Structure et diversité floristique des îlots de forets riveraines } \\
\hline $\mathrm{mPh}$ & GC & Loganiaceae & Anthocleista vogelii Planch. & 1 & 1 & \\
\hline MPh & GC & Moraceae & Antiaris toxicaria Lesch. ssp. welwitschii (Engl.) C & & 1 & 1 \\
\hline $\mathrm{mph}$ & SG & Euphorbiaceae & Antidesma venosum E. Mey. ex Tull. & 1 & 1 & \\
\hline $\mathrm{mph}$ & GC & Sapindaceae & Aphania senegalensis (Juss. ex Poir.) Radlk. & & 1 & 1 \\
\hline $\mathrm{mPh}$ & PRG & Meliaceae & Azadirachta indica A. Juss. & 1 & & \\
\hline $\mathrm{mPh}$ & As & Poaceae & Bambusa vulgaris Scharad. ex Wendel. & 1 & & 1 \\
\hline $\mathrm{mph}$ & GE & Passifloraceae & Barteria nigritana Hook.f. & & 1 & \\
\hline $\mathrm{mPh}$ & SG & Leguminosae-Caesalpinioideae & Berlinia grandiflora (Vahl) Hutch. \& Dalziel & & & 1 \\
\hline nph & GC & Apocynaceae & Callichilia barteri (Hook.f.)Stapf & & 1 & 1 \\
\hline nph & GC & Ochnaceae & Capylospermum glaberrimum (P. Beauv.) Farron & 1 & 1 & 1 \\
\hline $\mathrm{mph}$ & GC & Polygalaceae & Carpolobia lutea G.Don & & & 1 \\
\hline $\mathrm{mPh}$ & SG & Rhizophoraceae & Cassipourea congoensis R.Br. ex DC. & 1 & 1 & 1 \\
\hline MPh & PRG & Bombacaceae & Ceiba pentandra (L.) Gaertn & & 1 & 1 \\
\hline $\mathrm{mph}$ & GC & Celtidaceae & Celtiis prantlii Priemer ex Engl. & & 1 & \\
\hline nph & GC & Rubiaceae & Chassalia kolly (Schumach.) Hepper & & 1 & 1 \\
\hline $\mathrm{mPh}$ & GC & Sterculiaceae & Cola gigantea A. Chev. var. gigantea & & 1 & \\
\hline $\mathrm{mPh}$ & SG & Sterculiaceae & Cola laurifolia Mast. & & 1 & 1 \\
\hline $\mathrm{mph}$ & GC & Sterculiaceae & Cola millenii K.Schum. & & 1 & \\
\hline $\mathrm{mph}$ & GC & Connaraceae & Connarus africanus Lam. & & 1 & 1 \\
\hline $\mathrm{mph}$ & GC & Rubiaceae & Cuviera macroura K.Schum. & & 1 & \\
\hline $\mathrm{mPh}$ & GC & Leguminosae-Caesalpinioideae & Cynometra vogelii Hook.f. & & & 1 \\
\hline Lmph & GE & Leguminosae-Papilionoideae & Dalbergia lactea Vatke & & 1 & \\
\hline nph & GC & Sapindaceae & Deinbolia pinnata (Poir.) Schumach .\& Thonn. & & 1 & 1 \\
\hline $\mathrm{mPh}$ & GC & Leguminosae-Caesalpinioideae & Dialium guineense Willd. & & 1 & 1 \\
\hline $\mathrm{mph}$ & GC & Dracaenaceae & Dracaena arborea (Willd.) Link & & 1 & \\
\hline $\mathrm{mph}$ & GC & Euphorbiaceae & Drypetes floribunda (Müll.Arg.) Hutch. & & & 1 \\
\hline $\mathrm{mPh}$ & GC & Arecaceae & Elaeis guineensis Jacq. & 1 & & 1 \\
\hline $\mathrm{mph}$ & GC & Moraceae & Ficus lutea Vahl & 1 & 1 & 1 \\
\hline $\mathrm{mPh}$ & GC & Moraceae & Ficus vogeliana (Miq.) Miq. & 1 & & \\
\hline nph & PRG & Euphorbiaceae & Flueggea virosa (Roxb. ex Willd.) Voigt & 1 & & \\
\hline $\mathrm{mPh}$ & GC & Euphorbiaceae & Hura crepitans L. & 1 & 1 & \\
\hline Lmph & GC & Rubiaceae & Keetia multiflora (Schumach. \& Thonn.) Bridson & & 1 & 1 \\
\hline Lmph & GC & Apocynaceae & Landolphia togolana (Hallier f.) Pichon & & 1 & \\
\hline $\mathrm{mPh}$ & GC & Anacardiaceae & Lannea nigritana (Scott-Elliot) Keay & & 1 & \\
\hline $\mathrm{mph}$ & GC & Sapindaceae & Lecaniodiscus cupanioides Planch. ex Benth. & & 1 & 1 \\
\hline nph & SG & Rubiaceae & Macrosphyra longistyla (DC.) Hiern & 1 & & \\
\hline Lnph & GC & Melastomataceae & Memecylon afzelii G. Don. & & 1 & \\
\hline $\mathrm{mph}$ & GC & Leguminosae-Papilionoideae & Millettia griffoniana Baill. & & 1 & 1 \\
\hline $\mathrm{mph}$ & GC & Leguminosae-Papilionoideae & Millettia thonningii (Schumach. \& Thonn.) Baker & & & 1 \\
\hline nph & PRG & Leguminosae-Mimosoideae & Mimosa pigra L. & 1 & & \\
\hline $\mathrm{mph}$ & SZ & Sapotaceae & Mimusops kummel Bruce ex A.DC. & 1 & 1 & \\
\hline $\mathrm{mph}$ & SG & Rubiaceae & Mitragyna inermis (Willd.) Kuntze & 1 & & \\
\hline $\mathrm{mph}$ & SG & Rubiaceae & Morelia senegalensis A. Rich. ex DC. & 1 & 1 & \\
\hline
\end{tabular}




\begin{tabular}{|c|c|c|c|c|c|c|}
\hline \multicolumn{3}{|c|}{$\begin{array}{l}\text { Adjakpa et al. J. Appl. Biosci. } 2013 . \\
\text { communautaires de la vallée de Sô du Benir }\end{array}$} & \multicolumn{4}{|c|}{ Structure et diversité floristique des îlots de forets riveraines } \\
\hline $\mathrm{mph}$ & GC & Lecythidaceae & Napoleonaea vogelii Hook.\& Planch. & 1 & 1 & 1 \\
\hline $\mathrm{mPh}$ & GC & Bignoniaceae & Newbouldia laevis (P. Beauv.) Seem. ex Bureau & 1 & 1 & \\
\hline $\mathrm{mph}$ & SZ & Flacourtiaceae & Oncoba spinosa Forssk. & 1 & & \\
\hline $\mathrm{mph}$ & GC & Sapindaceae & Pancovia bijuga Willd. & & & 1 \\
\hline MPh & SG & Chrysobalanaceae & Parinari congensis F. Didr & & & 1 \\
\hline $\mathrm{mPh}$ & SZ & Leguminosae-Mimosoideae & Parkia biglobosa (Jacq.) R.Br. ex Benth. & 1 & & \\
\hline Lmph & PRG & Sapindaceae & Paullinia pinnata L. & & 1 & \\
\hline nph & PRG & Phytolacaceae & Petiveria alliacaea (Lam.) H. Walt. & & 1 & 1 \\
\hline $\mathrm{mph}$ & PRG & Myrtaceae & Psidium guajava $\mathrm{L}$. & 1 & & \\
\hline nph & $S G$ & Rubiaceae & Psychotria psychotrioides (DC.) Roberty & & & 1 \\
\hline nph & SG & Rubiaceae & Psychotria vogeliana Benth. & & 1 & 1 \\
\hline $\mathrm{mPh}$ & SG & Leguminosae-Papilionoideae & Pterocarpus santalinoides L'Hér. ex DC. & 1 & 1 & 1 \\
\hline $\mathrm{mph}$ & GC & Apocynaceae & Rauvolfia vomitoria Afzel. & & 1 & 1 \\
\hline nph & GC & Connaraceae & Rourea coccinea (Thom.ex Schumach. ) Benth. & 1 & & \\
\hline Lmph & GC & Apocynaceae & Saba comorensis (Bojer) Pichon & & 1 & \\
\hline Lmph & PRG & Rubiaceae & Sarcocephalus latifolius (Sm.) E.A. Bruce & & & 1 \\
\hline Lmph & GC & Anacardiaceae & Sorindeia grandifolia Engl. & & 1 & 1 \\
\hline $\mathrm{LmPh}$ & GC & Loganiaceae & Strychnos congolana Gilg. & & & 1 \\
\hline $\mathrm{mph}$ & GC & Sapotaceae & Synsepalum brevipes (Baker) T.D. Penn. & & 1 & \\
\hline $\mathrm{mPh}$ & PRG & Verbenaceae & Tectona grandis L.f. & 1 & & \\
\hline $\mathrm{mph}$ & GC & Meliaceae & Trichilia prieuriana A. Juss. & 1 & 1 & 1 \\
\hline Lmph & GC & Annonaceae & Uvaria chamae P. Beauv & 1 & 1 & \\
\hline $\mathrm{mPh}$ & SZ & Verbenaceae & Vitex doniana Sweet & 1 & 1 & 1 \\
\hline $\mathrm{mPh}$ & GO & Verbenaceae & Vitex ferrunigena Schumach. ex Thonn. & & 1 & \\
\hline Lmph & GC & Melastomataceae & Warneckea memecyloides (Benth.) Exell & & & 1 \\
\hline $\mathrm{mPh}$ & SG & Rutaceae & Zanthoxylum zanthoxyloides (Lam.) Zepernick \& Timler & 1 & & 1 \\
\hline
\end{tabular}

TB : Types Biologiques ( $\mathrm{L}$ : liane, MPh : mégaphanérophyte, $\mathrm{mPh}$ : mésophanérophyte, mph : microphanérophyte, nph: nanophanérophyte) TP: Types Phytogéographiques (GC: guinéo-congolais, GE : guinéo-oriental, SG : soudano-guinéen, $S Z$ : soudano-zambésien, PRG : plurirégional). 